\title{
El efecto de la fatiga cíclica sobre los pilares de implantes dentales
}

\section{The effect of the cyclic fatigue in dental implant abutments}

\author{
A. Haidar Wehbe*, N. Matos Garrido**, J. M. Ayllón Guerola***, \\ J. Moreno Muñoz**, E. Nuñez Marquez** , E. Velasco Ortega****
}

\section{RESUMEN}

Introducción. La conexión implante-pilar ha sido sugerida como crucial para el éxito a largo plazo de las restauraciones sobre implantes oseointegración y para prevenir futuras complicaciones biológicas y mecánicas. El objetivo de este estudio fué evaluar la influencia del test de fatiga cíclica en el comportamiento de las conexiones internas implante-pilar.

Métodos. 36 pilares mutiposición de implantes de titanio de conexión interna fueron divididos en 2 grupos: 18 pilares slim o curvos y 10 pilares rectos con diferentes alturas. Los pilares fueron apretados con una llave de torque a $30 \mathrm{Ncm}$. Una carga cíclica entre $175 \mathrm{~N}$ y $100 \mathrm{~N}$ fué aplicada con $30^{\circ}$ de inclinación axial al sistema de implantes durante 5 millones de ciclos.

Resultados. Los tests biomecánicos muestran una fractura de los implantes y de los tornillos de retención a una carga límite de $100 \mathrm{~N}$ de los pilares slim curvos y de $130 \mathrm{~N}$ en los pilares rectos. La fracturas aparecieron en la zona de unión entre el cuello y el cuerpo del implante y en los tornillos protéscios. No se han encontrado fracturas en los pilares multiposición.

Conclusiones. Este estudio indican que los tests de fatiga cíclica son importantes para analizar la respuesta biomecánica de los diferentes pilares en las conexiones implante-pilar de los sistemas de implantes.

PALABRAS CLAVE: Implantes dentales, conexión implant-pilar, carga cíclica, test de fatiga, biomecánica, implantología oral.

\begin{abstract}
Introduction. The connection implant-abutment has been suggested to be crucial for the long-term success of implant restorations and to prevent future biological and mechanic complications. The aim of this study was to evaluate the influence of fatigue cyclic test in the behavior of internal connection implant-abutments. Methods. Thirty six titanium abutments of internal connection implants were divided in two groups: 18 slim and 18 right multiunit abutments with different length. Abutments were tightened to $30 \mathrm{Ncm}$ with a torque controller. A cyclic load between $175 \mathrm{~N}$ and $100 \mathrm{~N}$ according to different implant abutments at a 30-degree angle to the long axis was applied to the implants for a 5 million cycles.

Results. Biomechanical testing showed implant and screw retention fracture in a limit load of $100 \mathrm{~N}$ of slim implant abutments and $130 \mathrm{~N}$ of right implant abutments. Fracture cracks were located in the area between neck and body of implants and screw retention. No abutment fractures were found.

* $\quad$ Licenciado en Odontología. Universidad Alfonso X el Sabio de Madrid. Máster de Implantología Oral. Universidad de Sevilla.

* $\quad$ Profesor del Máster de Implantología Oral. Facultad de Odontología. Universidad de Sevilla.

*** Departamento de Ingeniería Mecánica. Escuela Superior de Ingenieros Industriales. Universidad de Sevilla.

**** Profesor Titular de Odontología Integrada de Adultos y Gerodontología. Director del Máster de Implantología Oral. Facultad de Odontología. Universidad de Sevilla.
\end{abstract}


Conclusions. This study indicate that fatigue cyclic test are very important to analize the biomechanical behavior of different abutments in connection implant-abutment of implants systems.

KEY WORDS: Dental implants, implant-abutment connection, cyclic loading, fatigue test, biomechanics, implant dentistry.

Fecha de recepción: 14 de marzo de 2020

Fecha de aceptación: 21 de marzo de 2020

A. Haidar Wehbe, N. Matos Garrido, J. M. Ayllón Guerola, J. Moreno Muñoz, E. Nuñez Marquez, E. Velasco Ortega. El efecto de la fatiga cíclica sobre los pilares de implantes dentales. 2020; 36, (2): 89-97.

\section{INTRODUCCIÓN}

La existencia de la unión implante-pilar es uno de los factores más importantes que contribuye al éxito del tratamiento con implantes en los pacientes con pérdida parcial y/o total de su dentición natural. Los sistemas de implantes actuales suelen presentar dos componentes, el implante y el pilar protésico. El primer componente es insertado en el hueso alveolar durante la fase quirúrgica y el segundo componente se fija al implante durante la fase prostodóncica para soportar la prótesis correspondiente. Como el éxito de las restauraciones con implantes se basa en conseguir y mantener la oseointegración, es esencial que el implante posea un ajuste preciso, desde un punto de vista biomecánico, con su respectivo pilar para su carga funcional ${ }^{1}$.

Durante la masticación, el implante dental interactúa con las fuerzas oclusales que influyen negativamente sobre la unión implante-pilar. Estas fuerzas oclusales no suelen ser paralelas al eje axial del implante, sino que adquieren un eje tangencial u horizontal, generando un estrés biomecánico sobre la interfase hueso-implante, y además sobre la unión implante-pilar que debe soportarlo durante un largo periodo de tiempo ${ }^{2}$. Desde un punto de vista biomecánico, estas fuerzas oclusales desfavorables pueden general complicaciones en la unión de la prótesis con el implante, comenzando con la deformación de los materiales y continuando con la pérdida o fractura de los tornillos protésicos, movilidad o fractura de los de los pilares, e incluso fractura de los implantes ${ }^{2-3}$.

La mayoría de los sistemas de implantes suelen presentar una conexión protésica externa o interna para su unión con el correspondiente pilar protésico. Ambos tipos de conexiones dependen de su ajuste preciso con los pilares correspondientes y de su respuesta biomecánica estática y dinámica a la carga funcional ${ }^{4-5}$. En este sentido, el diseño macroscópico, el tamaño y la angulación de los implantes, así como de los pilares y el tipo y desarrollo de la conexión implante-pilar influyen de forma decisiva en su comportamiento biomecánico ${ }^{6-7}$.

Además, es importante valorar también las consecuencias biológicas en los tejidos periimplantarios del estrés excesivo y del desajuste o movilidad de la conexión implante-pilar. En este sentido, las restauraciones sobre implantes soportan las fuerzas masticatorias en un ambiente oral contaminado que afecta a la superficie del implante en su conexión con el pilar protésico ${ }^{8}$. De hecho, las fuerzas oclusales excesivas pueden contribuir a la creación de un microgap o espacio en la unión entre la superficie del implante y del pilar protésico que puede originar inflamación de los tejidos blandos y una progresiva pérdida marginal ósea ${ }^{9}$.

El objetivo del test de fatiga o carga cíclica es analizar la respuesta biomecánica de los diferentes componentes (implantes, pilares y tornillos) previamente a su aplicación clínica de una forma dinámica ${ }^{10}$. En este sentido, el desarrollo actual de la implantología actual supone la constante introducción de nuevos materiales y diseños que deben ser evaluados en estudios biomecánicos experimentales. Los diferentes sistemas de implantes deben ser evaluados para evitar o disminuir aquellas complicaciones derivadas del complejo implante-pilar durante el largo periodo de actividad clínica en los pacientes, lo que indica el gran reto que representa para inicialmente para los fabricantes y posteriormente para los clínicos ${ }^{11-13}$.

La presente investigación experimental tiene el objetivo de evaluar el efecto de la fatiga cíclica 
en pilares intermedios y de carga inmediata en implantes de conexión interna.

\section{MATERIAL Y MÉTODOS}

El presente estudio de fatiga cíclica de pilares protésicos sobre implantes dentales se ha realizado en el Departamento de Ingeniería Mecánica en la Escuela Superior de Ingenieros Industriales de la Universidad de Sevilla.

\section{Material}

En el presente estudio se han utilizado implantes dentales de conexión interna Surgimplant IPX ® (ref. IPX-03512) fabricado por Galimplant (Sarria, España). La plataforma del implante presenta un alojamiento hexagonal que permite la aplicación del par para la inserción del implante en el hueso fresado y además permite la retención antirrotacional de la prótesis. El diseño de este implante presenta una rosca con una morfología autorroscante (Figura 1). El material en el que se confeccionan estos implantes es titanio grado IV de acuerdo a la norma ISO- 5832-3:1999 ${ }^{14}$.

Los implantes Surgimplant IPX presentan su superficie tratada mediante un proceso de arenado con alúmina y grabado con ácidos para incrementar su rugosidad superficial. Dicho tratamiento ha sido desarrollado para mejorar las condiciones de oseointegración del implante en el hueso. Se extiende desde

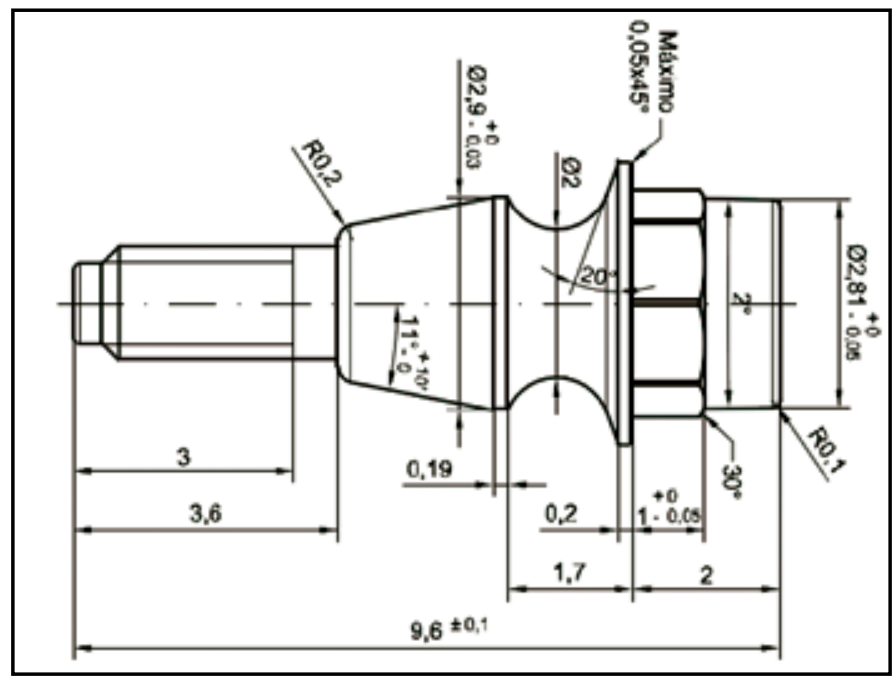

la plataforma hasta la zona apical del mismo.

Se ha seleccionado el diseño de cuerpo de implante de 3,5x12 mm (ref. IPX 3512), ya que es el menos resistente de todas las dimensiones del sistema Surgimplant IPX grado IV. En total se han ensayado un total de 9 muestras.

La parte conectora directa al cuerpo del implante se realiza mediante pilares intermedios multi-posición monoblock slim con curvatura (ref. MUSLA ®) o recto con dos piezas (ref. MUSA (B) (Figura 2). Estos pilares presentan en su parte superior pueden conectar con el segundo componente del aditamento, denominado pilar de carga inmediata.

El otro elemento de la parte conectora es el pilar de carga inmediata (ref. PTIMUA®). Este pilar presenta una parte superior donde se cementa la rehabilitación protésica. Este pilar presenta en su base un saliente con una cavidad octogonal que se encaja en el saliente existente en el pilar multi-posición, siendo su función la de evitar la rotación del elemento protésico respecto al implante.

Los pilares multiposición slim MUSLA se presentan con 4 diferentes alturas de 2, 3,4 y $6 \mathrm{~mm}$. Los pilares multiposición rectos MUSA se presentan con 5 diferentes alturas de 1,2,3,4 y $5 \mathrm{~mm}$. Un total de 9 muestras han sido ensayadas de cada tipo de pilar multiposición, seleccionado las alturas mayores y menores de cada tipo (MUSLA de 2 y $6 \mathrm{~mm}$ ) (MUSA de 1 y $5 \mathrm{~mm}$ ).

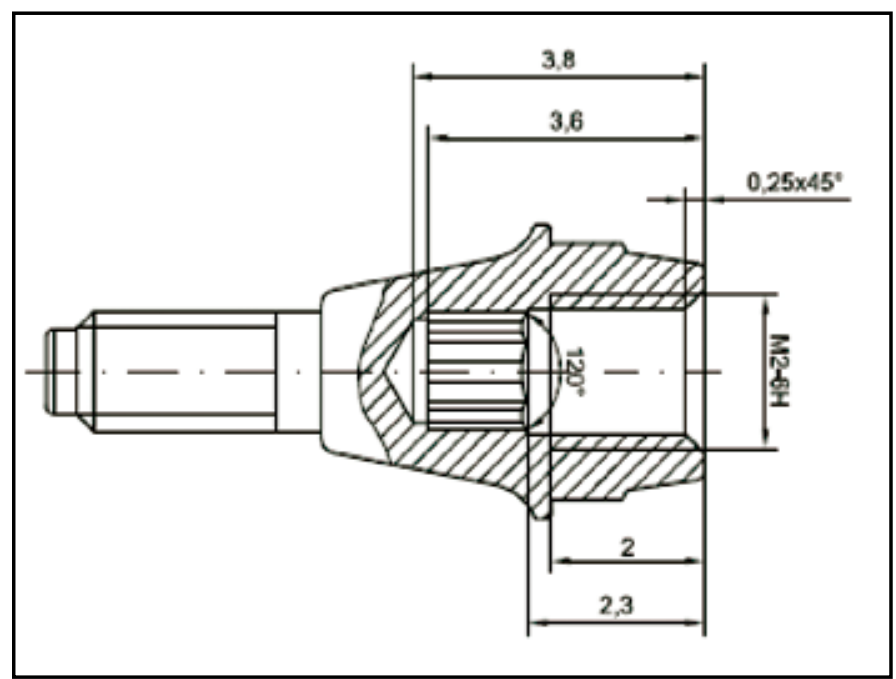

Figura 2. Descripción geométrica de los pilares multi-posición slim MUSLA y rectos MUSA. 


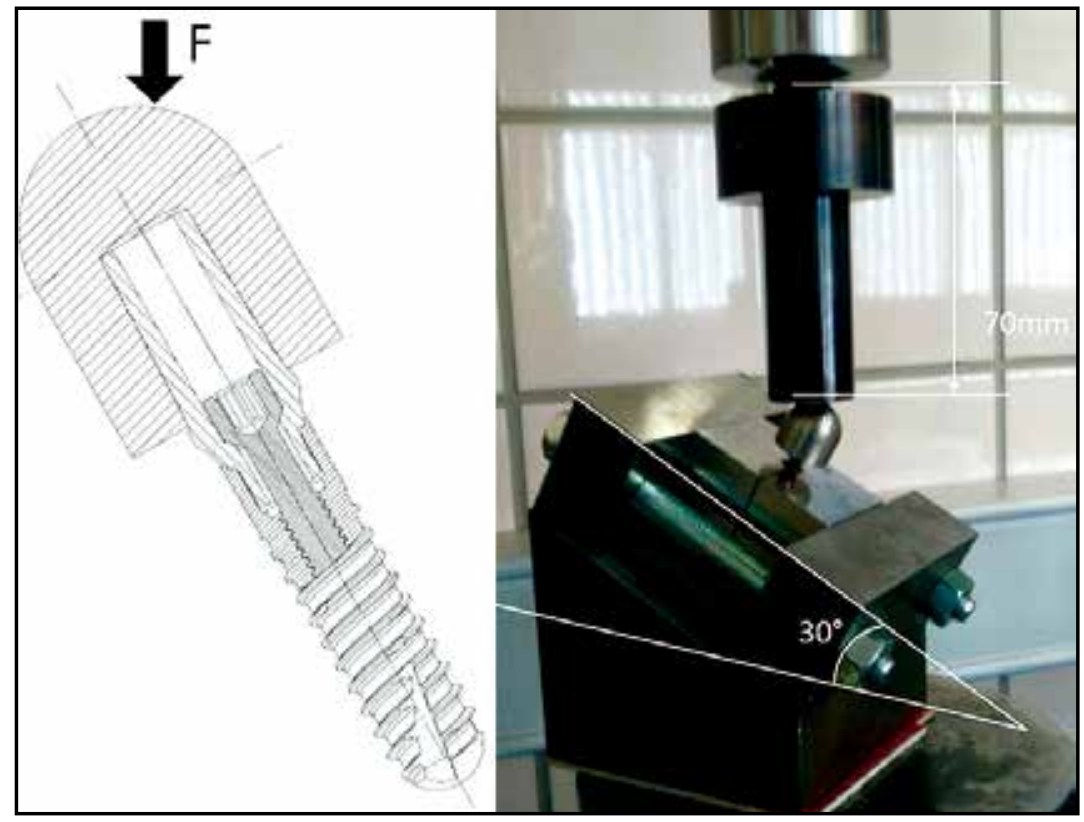

Figura 3. Configuración del ensayo y dispositivo para el test de fatiga cíclica.

Se emplea una llave dinamométrica CA DIN proporcionada por el fabricante, aplicando un par de apriete de $30 \mathrm{Ncm}$ para unir el pilar multi-posición al cuerpo del implante, así como para unir los dos pilares mediante el tornillo de retención.

\section{MÉTodos}

Para la realización de los ensayos se han empleado los equipos, máquina universal de ensayos mecánicos MTS $858 \mathrm{Mi}$ niBionix y célula de carga MTS 661.19F01 de $5 \mathrm{kN}$ con los que se cumplen los requisitos sanitarios y los especificados en la norma UNE-EN ISO 14801:2017 ${ }^{15}$.Todos los equipos de medida emplea-

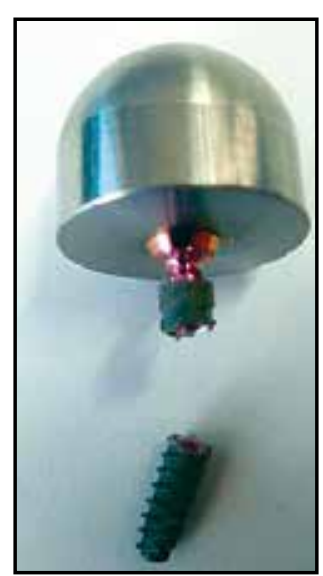

Figura 4. Modo de fallo del sistema de implantes. dos han sido calibrados según normas por empresas certificadas y tienen el certificado de calibración vigente Norma ISO 9001:2015 ${ }^{16}$.

Los ensayos se atienen en todo momento a lo dispuesto en la norma UNE-EN ISO 14801:2017 15. La Figura 3 muestra un esquema del montaje del implante en un dispositivo de anclaje fijo. Para la realización de las pruebas, el implante se coloca en un casquillo de cobre, que, al ser comprimido por un sistema de mordazas, se deforma plásticamente, de manera que produce un apriete uniforme sobre el implante. El módulo elástico del cobre es cercano a los $100 \mathrm{GPa}$, valor superior al que establece como mínimo la norma UNE-EN ISO 14801:2017 ${ }^{15}$ y un límite elástico menor a los $100 \mathrm{MPa}$, lo que es inferior al $12 \%$ del límite elástico del material del implante (Titanio Grado IV), lo que garantiza que la presión del cobre sobre la muestra no produce una deformación plástica.

El sistema de prensa y las mordazas están montados sobre un soporte con un plano mecanizado a $30^{\circ}$ respecto a la horizontal, con las tolerancias de fabricación, que son inferiores a un minuto. Este sistema, presenta unas guías laterales que garantizan el paralelismo entre las partes fijas y móviles del mismo.

Por otro lado, la fijación del implante en el casquillo, así como el diseño y colocación del miembro hemisférico de carga se han realizado de forma que se garantice que la distancia entre el centro del miembro hemisférico y el punto de intersección del eje del implante y la cara plana superior del casquillo (l, según la norma ISO 14801:2017) 15 está dentro de las tolerancias permitidas: $11 \pm 0.5 \mathrm{~mm}$.

De acuerdo con la norma UNE-EN ISO 14801:2017 $7^{15}$, las cargas cíclicas aplicadas han oscilado siempre entre un valor máximo (Pmax) y un valor mínimo igual al $10 \%$ del máximo (Pmin $=0.1$ Pmax). La elección de los niveles de carga y del número de muestras a ensayar en cada nivel (ver apartado de resultados) se atiene al hecho observado experimentalmente de que la dispersión de los resultados es mayor a cargas bajas. En cualquier caso, se cumplen los requisitos impuestos por la norma UNE-EN ISO 14801:2017 ${ }^{15}$. La incertidumbre en la medida de la carga aplicada producida por la célula de carga correspondiente es menor de $5 \mathrm{~N}$, valor inferior al máximo permitido que es el $5 \%$ de la carga máxima aplicada. El nivel de carga máximo al que ha sido sometido el implante se encuentra en torno al $80 \%$ del valor de la carga de fallo del mismo, obtenido mediante un ensayo estático en las mismas condiciones geométricas que los ensayos de fatiga. La frecuencia de aplicación de la carga es $15 \mathrm{~Hz}$. Las condiciones ambientales de la zona donde se han realizado los ensayos, son un medio con aire, temperatura de $25^{\circ}$ y una humedad relativa de $60 \%$. 


\section{RESULTADOS}

Durante la realización del test de fatiga cíclica, con los implantes y pilares ensayados se ha observado un único parón de fractura del complejo implante-pilar de rotura, provocado por la iniciación y propagación de una grieta por fatiga. La fractura tiene lugar en el cuerpo de implante, a la altura del plano de sujeción, como puede observarse en la Figura 4. El tornillo de retención también resulta fracturado. Durante todo el ensayo no hubo ninguna fractura del pilar protésico de conexión interna y del tornillo de conexión. Tampoco hubo aflojamiento del tornillo de conexión.

Los resultados muestran los valores de las cargas aplicadas en cada ensayo, así como el número de ciclos que han soportado los implante con los pilares protésicos testados con el tornillo de conexión. Además se indica la localización del punto crítico de fallo y descripción del mismo. Tanto la localización como la descripción se representan mediante los códigos (L1 y D1). Se ha identificado el punto de fallo crítico y la localización de la iniciación del fallo. El fallo se definió como el límite elástico del material, deformación permanente o fractura de cualquier componente. L1 es el cuerpo del implante coincidente con el plano superior del casquillo de sujeción. D1: Fractura del cuerpo del implante.

Las tablas 1 y 2 resumen las cargas aplicadas, así como el número de ciclos hasta el fallo o la terminación del ensayo para los tipos de pilares testados slim MUSLA de $2 \mathrm{~mm}$ y rectos MUSA de $1 \mathrm{~mm}$. En las dos últimas columnas se indica la localización y descripción del punto crítico de fallo. En todos los ensayos se ha obtenido el mismo tipo de fractura localizada en el plano de sujeción del implante. Las muestras que no se han fracturado durante el ensayo pueden identificarse por el símbolo (--) en las columnas asociada al tipo y localización del fallo.

La curva de fatiga obtenida para el implante y los pilares multiposición puede observarse en la Figura 5. En los test de fatiga cíclica, la respuesta biomecánica del material es representada por la curva donde se expresa el número de ciclos para alcanzar el fallo y la magnitud de las fuerzas utilizadas. Los valores por debajo de la curva indican los implantes que no se han fracturado.Las muestras cuyo ensayo ha sido detenido sin que haya tenido

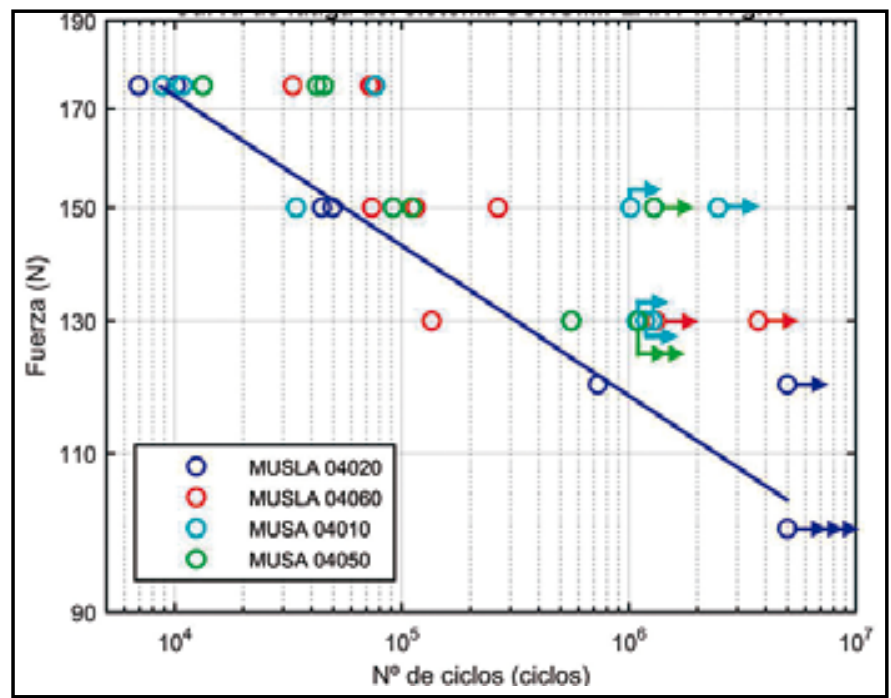

Figura 5. Curva de fatiga obtenida para el implante con los pilares rectos.

lugar sus fractura han sido marcadas con una flecha. Este diagrama representa el número de ciclos de carga que resiste cada muestra de implante (en escala logarítmica) y la correspondiente amplitud de carga (en escala lineal). Este diagrama de ciclos de carga muestra el módulo de elasticidad del implante a ensayar. Cada punto representa los resultados de una muestra de ensayo. Los implantes tienen una resistencia media a la fatiga como la mostrada en la curva de la Figura 5.

\section{DISCUSIÓN}

Los sistemas de implantes dentales con sus diversos componentes quirúrgicos (implantes) y protésicos (pilares y tornillos de conexión) deben estar diseñados y fabricados para asegurar su éxito en el medio oral y además poder soportar las cargas masticatorias durante un largo periodo de tiempo, lo que implica la necesidad de que los sistemas de implantes dentales estén elaborados de materiales con una resistencia adecuada que garantice su longevidad más de 20 años ${ }^{17-18}$.

En la actualidad, diversas estudios analizan los nuevos diseños de los sistemas de implantes y de los componentes protésicos para mejorar su respuesta biomecánica mediante protocolos de carga estática y cíclica que mejoren su aplicación clínica $^{3-7,10-12}$. Los estudios experimentales en laboratorio de ingeniería han demostrado que el éxito del tratamiento con implantes dentales está relacionado con su resistencia a las fuerzas oclu- 
AVANCES EN ODONTOESTOMATOLOGÍA

Vol. 36 - No 2 - 2020 Especial Monográfico IMPLANTOLOGÍA Oral

sales excesivas más desfavorables ${ }^{18-19}$.

El diseño macroscópico (incluyendo su diámetro y longitud) de los componentes de los sistemas de implantes está relacionado con la distribución de las fuerzas que se generan durante la masticación o el contacto oclusal repetitivo (bruxismo) al hueso periimplantario a través del complejo implante-pilar. Por tanto, la elección de la macrogeometría del sistema de implante puede prevenir problemas derivados de la sobrecarga oclusal como la pérdida ósea marginal, la movilidad de los pilares y de la prótesis, así como la fractura de cualquier componente (tornillo protésico, pilar e implante) ${ }^{13,20-21}$.

El presente test experimental de fatiga o carga cíclica constituye un método eficaz para determinar la integridad de los componentes de los sistemas de implantes (implantes, pilares y tornillos de conexión) con el objetivo de investigar y desarrollar nuevos aditamentos protésicos para los sistemas de implantes. Este tipo de test es reproducible y permite analizar, comparativamente, la respuesta biomecánica de diferentes diseños de los elementos del complejo pilar-implante bajo condiciones estandarizadas de carga cíclica ${ }^{6}$.

En este sentido, el presente estudio representa una investigación experimental sobre la resistencia de materiales dentales para implantología oral para su posterior aplicación clínica. De hecho, el estudio valora la respuesta a la fatiga cíclica de dos pilares intermedios multiposición con curvadura o slim y rectos para implantes de conexión interna.

Los aditamentos o pilares slim MUSLA (Figura 2) se han seleccionado para los ensayos porque, debido a su geometría curva más estrecha, puede ser considerado el más débil desde el punto de vista de la fatiga según las directrices de ensayo marcadas por la norma UNE-EN ISO 14801:2017 ${ }^{15}$. Sin embargo, la elección del caso más desfavorable para los ensayos no es sencilla desde un punto de vista biomecánico teórico.

En concreto, el pilar mutliposición MUSLA (Figura 2) ha sido seleccionado porque se sospecha que la zona critica más estrecha pudiera ser una zona de iniciación de grietas por fatiga debido a que el radio de curvatura hace que se produzca el fenómeno de concentración de tensión (la tensión se incrementa

\begin{tabular}{|c|c|c|c|c|c|}
\hline \multirow{2}{*}{ Muestra } & \multirow{2}{*}{$\begin{array}{c}\text { Max } \\
\text { Carga (N) }\end{array}$} & \multirow{2}{*}{$\begin{array}{c}\text { Min } \\
\text { Carga } \\
(\mathbf{N})\end{array}$} & \multirow{2}{*}{$\begin{array}{c}\text { Vida } \\
\text { (Ciclos) }\end{array}$} & \multicolumn{2}{|c|}{ Fallo } \\
\cline { 5 - 6 } & & & Localización & Descripción \\
\hline 1 & 175 & 17.5 & 10148 & L1 & D1 \\
\hline 2 & 175 & 17.5 & 6920 & L1 & D1 \\
\hline 3 & 150 & 15 & 49605 & L1 & D1 \\
\hline 4 & 150 & 15 & 44488 & L1 & D1 \\
\hline 5 & 120 & 12 & $5 \mathrm{e} 6$ & -- & -- \\
\hline 6 & 120 & 12 & 729426 & L. & D1 \\
\hline 7 & 100 & 10 & $5 \mathrm{e} 6$ & -- & -- \\
\hline 8 & 100 & 10 & $5 \mathrm{e} 6$ & -- & -- \\
\hline 9 & 100 & 10 & $5 \mathrm{e} 6$ & - & - \\
\hline
\end{tabular}

Tabla 1. Valores obtenidos, con todas las cargas aplicadas que han soportado los implantes Surgimplant IPX y pilares multiposición slim MUSLA de $2 \mathrm{~mm}$.

\begin{tabular}{|c|c|c|c|c|c|}
\hline \multirow{2}{*}{ Muestra } & \multirow{2}{*}{$\begin{array}{l}\text { Máx } \\
\text { Carga } \\
\text { (N) }\end{array}$} & \multirow{2}{*}{$\begin{array}{l}\text { Min } \\
\text { Carga } \\
\text { (N) }\end{array}$} & \multirow{2}{*}{$\begin{array}{l}\text { Vida } \\
\text { (Ciclos) }\end{array}$} & \multicolumn{2}{|c|}{ Fallo } \\
\hline & & & & Localización & Descripción \\
\hline 1 & 175 & 17.5 & 76466 & L1 & D1 \\
\hline 2 & 175 & 17.5 & 10837 & L1 & D1 \\
\hline 3 & 175 & 17.5 & 8825 & LI & D1 \\
\hline 4 & 150 & 15 & 34270 & L1 & D1 \\
\hline 5 & 150 & 15 & 2468434 & - & - \\
\hline 6 & 150 & 15 & 1017729 & -- & - \\
\hline 7 & 130 & 13 & 1183000 & - & - \\
\hline 8 & 130 & 13 & 1080089 & -- & -- \\
\hline 9 & 130 & 13 & 1274476 & L1 & D1 \\
\hline
\end{tabular}

Tabla 2. Valores obtenidos, con todas las cargas aplicadas que han soportado los implantes Surgimplant IPX y pilares multiposición rectos MUSA de $1 \mathrm{~mm}$.

en la zona del radio de curvatura). Además, se suma al hecho de que la sección del pilar en esa zona es muy pequeña, por presentar un alojamiento interior hexagonal para la aplicación del torque durante su inserción en el implante ${ }^{18}$.

Por este motivo se ha decidido realizar ensayos adicionales con otros aditamentos protésicos (pilares rectos MUSA)(Figura 2) para los que, desde un punto de vista teórico, no es posible justificar de una manera concluyente que su resistencia a fatiga es superior (o al menos igual) a la del pilar slim MUSLA ensayado. En este sentido, el presente estudio de fatiga cíclica demuestra que ambos pilares multiposición, curvos y rectos, no presentan una reducción de la resistencia a fatiga del sistema, porque en todos los ensayos el punto de fallo ha sido siempre el cuerpo del implante y el tornillo de conexión (Tablas 1 y 2). 
Entre los objetivos de los tests de fatiga cíclica se describen el análisis estandarizado de los diferentes mecanismos que aseguren la conexión implante-pilar. El análisis de las fuerzas de cargas axiales y tangenciales que influyen en la fatiga de estos materiales es importante porque pueden producir un daño progresivo, localizado y permanente de la estructura de un material (ej. implante, pilar, tornillo de conexión) sometido a repetidos ciclos de tensión 4,19. Desde un punto de vista biomecánico, las posibles complicaciones de los componentes de los sistemas de implantes como puede ser la fractura del cuerpo de un implante y/o de los pilares y tornillos protésicos depende por un lado de factores extrínsecos, o sea las fuerzas oclusales que actúan sobre la estructura macroscópica de estos elementos quirúrgicos y prostodóncicos y de factores intrínsecos resultantes de su módulo de elasticidad o de su resistencia estructural ${ }^{22-23}$.

Estos ensayos de fatiga se realizan mediante una carga cíclica variable de amplitud predeterminada y se registra el número de ciclos de carga hasta que ocurre un fallo. Las propiedades de fatiga de los ensayos se determinan por el número de muestras ensayadas. Experimentalmente, se utilizan fuerzas de carga axiales, pero aplicadas con un grado de inclinación, para simular fuerzas tangenciales, sobre el complejo implante-pilar evaluando su resistencia en los diferentes sistemas de implantes (Figura 3) 24. Este protocolo estandarizado de fatiga cíclica para estudios de sistemas de implantes dentales ha sido desarrollado por un consejo de expertos científicos e industriales de la Organization for International Standardization (ISO 14081) con unas recomendaciones para evaluar implantes dentales intraóseos transmucosos y autorizar su comercialización en Europa 24.

La precisión del ajuste de los componentes del complejo implante-pilar es fundamental para el éxito de su comportamiento biomecánico. El desajuste entre la plataforma del implante y el pilar de la prótesis origina un espacio, microgap, que puede dar lugar a fracturas del tornillo de conexión y otros componentes protéticos, fractura del implante, y distribución inadecuada de fuerzas al hueso, así como complicaciones biológicas relacionadas con la contaminación bacteriana (mucositis, periimplantitis) ${ }^{9-10,18}$.

Hay evidencia científica de la existencia de microgap en la conexión implante-pilar previamente a la carga funcional, que puede ser demostrada en imágenes de microscopia electrónica de barrido, y que se incrementa durante el test de fatiga cíclica lo que sugiere un problema importante de ajuste en la interfase entre el pilar y el implante 25 . Frecuentemente, la falta de precisión en el ajuste de la conexión implante-pilar puede ser originada por una deformación del material de titanio del implante como consecuencia de sobrecargas oclusales, fuerzas no axiales, colocación de restauraciones sin ajuste pasivo y apretamiento inadecuado del tornillo protésico ${ }^{26}$.

En el presente estudio, el torque utilizado en todas las conexiones implante-pilar ensayadas era de $30 \mathrm{Ncm}$ que constituye un torque de apretamiento adecuado, teniendo en cuenta que entre $25 \mathrm{Ncm}$ y $40 \mathrm{Ncm}$, el microscopio electrónico de barrido no demuestra daños en la morfología del tornillo protésico indicando que está por debajo del límite elástico del material ${ }^{19}$.

El tornillo de conexión protésica es un elemento importante en la unión implante-pilar, ya que su configuración macroscópica influye esencialmente en el ajuste del implante y el pilar, siendo un elemento esencial en la estabilidad a largo plazo de las restauración protésica sobre el implante ${ }^{27}$. La colocación del tornillo de conexión de forma adecuada uniendo el pilar y el implante y su posterior torque de fijación constituye un paso esencial para conseguir una buena respuesta biomecánica de todo el complejo implant-pilar, evitando problemas derivados de microgap, desajuste, movilidad y fractura de este componente protésico ${ }^{28-29}$.

El presente estudio de investigación experimental demuestra la importancia de realizar ensayos de fatiga cíclica para evaluar el comportamiento biomecánico de los componentes de los sistemas de implantes dentales con nuevos diseños y materiales previamente a su aplicación clínica ${ }^{3,10,13,24}$.

\section{CONCLUSIONES}

Los estudios de fatiga cíclica representa un método contrastado y aceptado internacionalmente para analizar la respuesta biomecánica de los diversos componentes de los sistemas de implantes dentales. Los estudios de fatiga cíclica permiten identificar la localización de los puntos críticos donde es posible la incidencia de fallos estructu- 
rales el límite elástico del material, con deformación permanente o fractura de cualquier componente del sistema de implantes.

\section{BIBLIOGRAFIA}

1. Graves CV, Harrel SK, Rossmann JA, Kerns D, Gonzalez JA, Kontogiorgos ED et al. The role of occlusion in the dental implant and peri-implant condition: a review. The Open Dent J 2016; 10:594-601.

2. Abichandani SJ, Bhojaraju N, Satyabodh Guttal S, Srilakshmi J. Implant protected occlusion: A comprehensive review. Eur J Prosth 2013; 1:29-36.

3. Velasco-Ortega E, Flichy-Fernández A, Punset M, Manero JM, Gil FJ. Fracture and fatigue of $\mathrm{CP}$ titanium narrow dental implants. New trends in order to improve the mechanical response. Materials 2019; 12: 3728.

4. Feitosa PP, de Lima AB, Silva-Concílio LR, Brandt WC, Claro Neves AC. Stability of external and internal implant connections after a fatigue test. Eur J Dent 2013;7:267-71.

5. Goiato MC, Pellizzer EP, Vivianne E. Is the internal connection more efficient than external connection inmechanical, biological, and esthetical point of views? A systematic review. Oral Maxillofac Surg 2015; 19: 229-242.

6. Wang K, Geng J, Jones D, Xu W. Comparison of the fracture resistance of dental implants with different abutment taper angles. Mater Sci Eng C 2016; 63:164-171.

7. Suzuki H, Hata Y, Watanabe F. Implant fracture under dynamic fatigue loading: influence of embedded angle and depth of implant. Odontology 2016; 104:357-362.

8. Shemtov-Yona K, Rittel D, Levin L, Machtei EE. The effect of oral-like environment on dental implants' fatigue performance. Clin Oral Impl Res 2014; 25:66-70.

9. Khongkhunthian P, Khongkhunthian S DDS, Weerawatprachya W, Pongpat K, Aunmeungtong W. Comparative study of torque resistan- ce and microgaps between a combined Octatorx-cone connection and an internal hexagon implant-abutment connection. J Prosthet Dent 2015;113:420-424.

10. Matos-Garrido N, Moreno-Silván M, Ayllón-Guerola JM, Jiménez-Guerra A, Ortiz-Garcia I, España-López A, Segura-Egea $\mathrm{JJ}$, Velasco-Ortega E. Influencia de la fatiga cíclica en las conexiones internas implante-pilar. Av Odontoestomatol 2017; 33:221-229.

11. Duan Y, Jason A Griggs JA. Effect of loading frequency on cyclic fatigue lifetime of a standard-diameter implant with an internal abutment connection. Dent Mater 2018; 34:17111716.

12. Yao KT, Kao HC, Cheng CK, Fang HW, Huang $\mathrm{CH}$, Hsu ML. Mechanical performance of conical implant-abutment connections under different cyclic loading conditions. J Mech Behav Biomed Mater 2019; 90:426-432.

13. Valente MLC, de Castro DT, Macedo AP, Shimano AC, dos Reis AC. Comparative analysis of stress in a new proposal of dental implants. Mater Sci Eng C 2017; 77:360-365.

14. Asociación Española de Normalización y Certificación. Evaluación preclínica de la biocompatibilidad de los productos sanitarios usados en odontología. UNE-EN ISO 7405. Madrid: Aenor. 1999.

15. Asociación Española de Normalización y Certificación. Ensayo de fatiga para implantes dentales endoóseos. UNE-EN ISO 14801. Madrid: Aenor. 2017.

16. Asociación Española de Normalización y Certificación. Sistemas de gestión de la calidad. Norma ISO 9001. Madrid: Aenor. 2015.

17. Faria ACL, Rodrigues RCS, Claro APRA, de Mattos MGC, Ribeiro RF. Wear resistance of experimental titanium alloys for dental applications. J Mech Behav Biomed Mater 2011; 4: 1873-1879.

18. Gil FJ, Herrero-Climent M, Lázaro P, Ríos JV. Implant-abutment connections: influence of the design on the microgap and their fatigue 
and fracture behavior of dental implants. J Mater Sci: Mater Med 2014; 25: 1825-1830.

19. Coray R, Zeltner M, Ozcan M. Fracture strength of implant abutments after fatigue testing: A systematic review and a meta-analysis. J Mechanic Behav Biomed Mater 2016; 62: 333346.

20. Ribeiro CG, Maia MLC, Scherrer SS, Cardoso AC, Wiskott HWA. Resistance of three implant-abutment interfaces o fatigue testing. J Appl Oral Sci 2011; 19: 413-420.

21. Shemtov-Yona K, Rittel D, Machtei EE, Levin L. Effect of dental implant diameter on fatigue performance. Part I: Mechanical behavior. Clin Impl Dent Relat Res 2014; 16:172-177.

22. Shemtov-Yona K, Rittel D, Levin L, Machtei EE. Effect of dental implant diameter on fatigue performance. Part II: Mechanical behavior. Clin Impl Dent Relat Res 2014; 16:178-184.

23. Asvanund $P$, Morgano M. Photoelastic stress analysis of external versus internal implant-abutment connections. J Prosthet Dent 2011;106:266-271.

24. Bacchi A, Consani RLX, Mesquita MF, dos Santos MBF. Stress distribution in fixed-partial prosthesis and peri-implant bone tissue with different framework materials and vertical misfit levels: a three-dimensional finite element analysis. J Oral Science 2013; 55: 239-245.

25. Marchetti E, Ratta S, Mummolo S, Tecco S, Pecci R, Bedini R, MS, Marzo G.Mechanical reliability evaluation of an oral implant-abutment system according to UNI EN ISO 14801 fatigue test protocol. Implant Dent 2016; 25:613-618.

26. Blum K, Wiest W, Fella C, Balles A, Dittmann $\mathrm{J}$, Rack A, et al. Fatigue induced changes in conical implant-abutment connections. Dent Mater 2015; 31:1415-1426.

27. Micarelli C, Canullo L, Glannello L. Implant-abutment connection deformation after prosthetic procedures: An in vitro study. Int J Prosthodont 2015; 28:282-286.

28. Kanneganti KC, Vinnakota DN, Pottem SR, Pulagam $M$. Comparative effect of implant-abutment connections, abutment angulations, and screw lengths on preloaded abutment screw using three-dimensional finite element analysis: An in vitro study. J Indian Prosthodont Soc 2018;18:161-167.

29. Xia D, Lin H, Yuan S, Bai W, Zheng G. Dynamic fatigue performance of implant abutment assemblies with different tightening torque values. BioMed Mater Eng 2014; 24: 2143 2149.

30. Paepoemsin T, Reichart PA, Chaijareenont $P$, Strietzel FP, Khongkhunthian P. Removal torque evaluation of three different abutment screws for single implant restorations after mechanical cyclic loading. Oral Implantol 2016; 9: 213-221.

\section{AUTOR DE CORRESPONDENCIA:}

Prof. Eugenio Velasco Ortega

Facultad de Odontología

C/ Avicena s/n

Tfno: 954 481132, e-mail: evelasco@us.es 41009 Sevilla. 